rh 19 Revue d'histoire du XIXe siècle

Société d'histoire de la révolution de 1848 et des

révolutions du XIXe siècle

$26 / 27 \mid 2003$

Varia

\title{
Sudhir Hazareesingh (dir.), The Jacobin Legacy in Modern France, Oxford, Oxford University Press, 2001, 244 p.
}

Philippe Darriulat

\section{OpenEdition}

Journals

Édition électronique

URL : https://journals.openedition.org/rh19/762

DOI : $10.4000 /$ rh19.762

ISSN : $1777-5329$

Éditeur

La Société de 1848

Édition imprimée

Date de publication : 1 décembre 2003

Pagination : 376-380

ISSN : 1265-1354

Référence électronique

Philippe Darriulat, « Sudhir Hazareesingh (dir.), The Jacobin Legacy in Modern France, Oxford, Oxford University Press, 2001, 244 p. », Revue d'histoire du XIXe siècle [En ligne], 26/27 | 2003, mis en ligne le 23 juin 2005, consulté le 28 février 2022. URL : http://journals.openedition.org/rh19/762 ; DOI : https:// doi.org/10.4000/rh19.762

Ce document a été généré automatiquement le 28 février 2022.

Tous droits réservés 


\title{
Sudhir Hazareesingh (dir.), The Jacobin Legacy in Modern France, Oxford, Oxford University Press, 2001, 244 p.
}

\author{
Philippe Darriulat
}

1 Ce recueil d'articles est publié par une équipe de chercheurs anglais et français, historiens mais aussi juristes ou spécialistes des sciences politiques, soucieux de rendre hommage aux travaux et à la carrière de Vincent Wright décédé le 8 juillet 1999. Sudhir Hazareesingh (Balliol College, Oxford), qui a coordonné ce travail, nous présente dans un premier article ( Vincent Wright and the Jacobin Legacy in Historical and Theorical Perspectives») la ligne éditoriale fixée aux rédacteurs des différentes contributions: examiner les formes post-révolutionnaires du jacobinisme comme modèle d'organisation de l'État.

2 Il s'agit, pour les contributeurs, de mettre en évidence comment le jacobinisme - au sens moderne du terme-indissolublement lié dans les esprits à la tradition républicaine, s'est construit en incorporant des éléments de l'absolutisme, des principes et des valeurs libérales et des héritages des deux bonapartismes. Une étude portant sur l'ensemble de la période contemporaine qui devrait, pense Sudhir Hazareesingh, permettre de mieux comprendre certaines contradictions de la société française actuelle.

Dans « La Guerre sainte : Debates about Just War among Republicans in the Nineteenth Century", Karma Nabulsi (Nuffield College, Oxford), cherche à inscrire dans une continuité l'attitude des républicains français vis-à-vis de la guerre telle qu'elle s'exprime lors du conflit de 1870. Pour l'auteur, c'est la génération de 1830 qui intègre la guerre dans la culture politique du parti républicain. Légitimée par le devoir de révolte contre la tyrannie elle devient, pour eux, un état permanent et en quelque sorte naturel jusqu'à l'instauration de la république. Il s'agit d'une « guerre sainte » unissant l'ensemble des peuples européens dans une même lutte dont les aspects militaires et 
politiques sont indissolublement liés : défaites et victoires ne pouvant s'expliquer que par le degré d'authenticité des valeurs animant les combattants. L'invasion étrangère étant considérée comme la forme la plus achevée du despotisme, les guerres de libération - celle des Polonais et des Italiens notamment - bénéficieraient de la plus forte légitimité, supérieure à celle d'une guerre française, notamment pendant le règne de Louis-Philippe. Une affirmation qui aurait peut-être eu besoin d'être nuancée ; les traités de 1815 étaient alors vécus comme une insoutenable atteinte à la souveraineté territoriale et nationale justifiant pleinement le déclenchement d'une offensive de la France pour retrouver ses « frontières naturelles".

4 Les sociétés républicaines, nationales mais surtout locales, jouaient un rôle primordial en développant, par leur propagande - brochures, journaux, chansons, toasts portés lors des banquets, etc. - une véritable "culture de la guerre " alimentée par les pratiques de la clandestinité (formes d'organisation, codes, signes de reconnaissance, etc.) La solidarité avec des peuples agressés représentait un argument essentiel pour appeler au combat (cf. le rôle de la question polonaise dans l'évolution d'Armand Carrel). C'est cette culture qui est en place et s'exprime au grand jour, lorsque éclate le conflit de 1870. L'auteur rejette en conséquence logiquement toute opposition radicale, sur l'attitude à adopter face au conflit, entre les différents courants républicains de 1870 - il développe cette idée à partir des exemples de Gambetta et Esquiros - insistant au contraire sur ce qui les unit dans une même vision d'une république guerrière. Une idée intéressante mais qui, là encore, mériterait d'être nuancée au regard des profondes évolutions et ruptures qui ont marqué le parti républicain pendant l'Empire. Il rejette aussi, pour les mêmes raisons, la distinction entre tradition républicaine et révolutionnaire du jacobinisme: les révolutionnaires ne voulaient pas uniquement renverser le pouvoir, ils voulaient aussi défendre la patrie contre l'envahisseur.

5 Sudhir Hazareesingh consacre son article aux médaillés de Sainte-Hélène : « Honorable and Honoured Citizens : War Veterans of the Revolutionary and Napoleonic Eras under the Second Empire ». Véritables héros des communautés villageoises, les titulaires de cette distinction fondée par décret du 22 août 1857 jouaient, notamment à l'échelle communale, un rôle déterminant dans la perpétuation de la légende napoléonienne. Souvent dépourvus de papiers prouvant leur statut, parfois illettrés, ils ne devaient compter que sur leur renommée locale et sur le soutien des autorités municipales pour faire valoir leurs droits. L'auteur, à partir notamment d'un riche travail sur les archives départementales (Bouches-du-Rhône, Hérault, Loire-Atlantique, Marne, Meuse, Nord, Rhône, Seine-Maritime, Somme, Var, Vaucluse, Yonne), s'intéresse tout particulièrement à la place qu'occupaient ces vétérans, entre 1858 et 1869, dans les fêtes du 15 août où leur présence donnait une dimension commémorative aux festivités. Leur place dans la procession des notables de la commune, les banquets donnés la plupart du temps en leur honneur à la fin de cette journée de fête - l'auteur récuse l'opposition entre un 15 août impérial chauvin et bigot et un 14 juillet de la III ${ }^{\mathrm{e}}$ République festif et joyeux - étaient conçus à la fois pour permettre l'identification de la communauté avec "ses" héros et favoriser la récupération par le régime de la légende impériale.

6 Très attachés à leur honneur, les médaillés défendaient scrupuleusement leur place dans ces commémorations. Ils permettaient d'y célébrer les aspects politiques et civiques de l'épopée bonapartiste, y compris au détriment des thèmes militaires, les sources faisant peu état de vétérans racontant leurs exploits. Ils développaient aussi de 
profonds réseaux de solidarité qui se manifestaient notamment lors des funérailles de l'un d'entre eux ou dans la fondation de mutuelles de secours.

7 Pour Sudhir Hazareesingh, il s'agit avant tout, à travers ces manifestations d'un rappel $\mathrm{du}$ rôle déterminant du "peuple» dans le mythe bonapartiste. Un peuple que symbolisaient les médaillés investis des qualités de la simplicité, du martyre, de la force morale et de la fierté. Une «noblesse de cœur » qui, fondée sur l'honneur, la vertu et l'effort, sublimait les différences sociales. Pour conclure cet article riche de nombreux exemples, où l'on s'étonne cependant de ne pas trouver de références aux travaux de Natalie Petiteau, l'auteur voit dans ce 15 août commémoratif la marque de la vivacité de l'idéologie bonapartiste et en même temps une préparation du 14 juillet républicain.

Jean-Pierre Machelon (Paris V), dans la continuité des travaux de Vincent Wright qui était fasciné par la stabilité du personnel administratif de la haute fonction publique de la France du XIX ${ }^{\mathrm{e}}$ siècle, s'intéresse aux préfets de la République, du 4 septembre à la déclaration de guerre ("The Prefect, Political Fuctionary of the Jacobin State: Permanences and Continuities 1870-1914»).

9 Tout en confirmant la tendance à la professionnalisation et à la stabilisation du corps préfectoral - le modèle napoléonien d'un corps discipliné, hiérarchisé et puissant est remplacé par des préfets prudents, flexibles, conservateurs dont l'influence reposait sur l'observation des contraintes administratives et politiques plus que sur les textes officiels - l'auteur insiste sur un certain nombre de continuités dans l'exercice de cette fonction. Le degré de changement qui intervient au début de la Troisième République dans les pratiques des préfets était extrêmement limité et ces derniers, sur le terrain, ne devaient pas sembler bien différents de ce qu'ils étaient sous le Second Empire. Organisateurs, par exemple, des élections ils ne revendiquent toujours pas la moindre autonomie vis-à-vis des responsables politiques et restent les «fonctionnaires politiques » par excellence. Une politisation qui ne peut être dissociée de l'état d'esprit « jacobin » alors dominant, ce refus de toute décentralisation ou de reconnaissance des pouvoirs municipaux (souvenir de la Commune).

10 En fait la professionnalisation du corps préfectoral ne remet pas en cause la relation de dépendance avec les gouvernants. Les nominations restent entièrement discrétionnaires, jusqu'en 1928 - jusqu'en 1911 pour les sous-préfets et secrétaires généraux de préfecture - le revers de la médaille étant l'absence de stabilité.

11 L'analyse sociologique du corps préfectoral nous montre un groupe plus provincial et moins bourgeois que les autres grands corps de l'État. Ils sont aussi souvent politisés (en 1901 40\% sont liés à une figure politique et 27\% engagés eux-mêmes dans la vie politique) ce qui fait d'eux de véritables agents électoraux du gouvernement qu'ils tiennent régulièrement informé des surveillances exercées sur les opposants. Souples, pas trop scrupuleux et fermes avec les adversaires du gouvernement, ils seraient les dignes successeurs des préfets de Napoléon I $^{\text {er }}$ et de Napoléon III. Y compris après 1879 ils doivent être entièrement dévoués au gouvernement, même si les limogeages motivés par des considérations purement politiques sont maintenant dénoncés comme arbitraires. En fait cette politisation du corps n'était incompatible ni avec un certain degré de continuité ni avec une professionnalisation. En affirmant que le préfet est d'abord et avant tout un serviteur de l'État, plus qu'un défenseur des intérêts politiques du pouvoir, on l'autorise, au nom d'un intérêt supérieur, à changer d'attitude selon les majorités. On peut alors distinguer deux types de préfets: les malins-ceux qui 
savaient interpréter le langage politique et adapter les directives gouvernementales aux contraintes locales - et les combatifs qui restent insérés dans des réseaux militants.

Olivier Ihl (IEP Grenoble), réfléchit sur l'importance des décorations dans les Etats européens et notamment dans la France post-révolutionnaire («Emulation through Decoration: A Science of Government?»). S'interrogeant sur l'apparente contradiction entre les principes égalitaires de la Révolution - la Législative avait supprimé le 17 juin 1790 toute une gamme de distinction, fidèle en cela aux principes établis par Montesquieu et Mirabeau - et l'utilisation massive par la République des décorations, il revient sur l'histoire des ordres et des distinctions dans la France médiévale. L'auteur constate une profonde évolution, amorcée dès le XVII ${ }^{\mathrm{e}}$ siècle, formulée au XVIII ${ }^{\mathrm{e}}$ siècle (Guiseppe Gorani et Jeremy Bentham), mais concrétisée après la Révolution, y compris pendant la Restauration et l'Empire, substituant à une "société d'honneur " une «bureaucratie des honneurs ». Ainsi, si les récompenses honorifiques ne représentent pas une survivance des Ordres d'Ancien Régime - elles ne donnent accès à aucun privilège elles témoignent d'une certaine continuité dans l'action et l'affirmation de l'État moderne.

13 Ce recueil contient aussi d'autres articles qui nous intéressent moins directement ici (Maurice Larkin, «Fraternity, Solidarity, Sociability: the Grass Roots of the Grand Orient de France 1900-1926»; Philip Nord, «Reform, Conservation, and Adaptation : Sciences-Po from the Popular Front to the Liberation »; Douglas Johnson, «General De Gaulle and the Restoration of the Republic»; Yves Mény, "The Republic and its Territory: The Persistence and the Adaptation of Founding Myths»; Dominique Schnapper, «Making Citizens in a Increasingly Complex Society: Jacobinism Revisited ») ainsi qu'une bibliographie complète des écrits de Vincent Wright.

$14 \mathrm{Au}$ total un ouvrage qui vise à démontrer une continuité, par-delà les différents régimes, des formes - la centralisation - comme des personnels de l'État français. Un travail stimulant qui utilise souvent, notamment pour notre XIX ${ }^{e}$ siècle, les outils des sciences politiques et dans lequel les spécialistes d'histoire politique pourront trouver des éléments de réflexion et de débat. 